\title{
Machine Learning Augmented Causal Inference To Estimate The Treatment Effect of Tranexamic Acid In Traumatic Brain Injury
}

Imke Meyer

School for Advanced Studies in the Social Sciences

Jean-Denis Moyer

Hôpital Beaujon, DMU PARABOL, University of Paris

Aliénor Dreyfus

Hôpital Beaujon, DMU PARABOL, University of Paris

Boutonnet Mathieu

Hôpital d'instruction des Armées Percy

Pierre-Julien Cungi

Hôpital d'Instruction des Armées Sainte-Anne

Arnaud Foucrier

Hôpital Beaujon, DMU PARABOL, University of Paris

Anatole Harrois

$\mathrm{CHU}$ de Bicêtre

Arthur James

PSL University, CNRS, Sorbonne University, Université de Paris

Jean-Pierre Nadal

School for Advanced Studies in the Social Sciences

Julie Josse

French Institute for Research in Computer Science and Automation

Tobias Gauss ( $\nabla$ tgauss@protonmail.com )

Hôpital Beaujon, DMU PARABOL, University of Paris

\section{Research Article}

Keywords: Causal inference, machine learning, trauma science, IPW, doubly robust, missing data

Posted Date: July 6th, 2021

DOI: https://doi.org/10.21203/rs.3.rs-600886/v1 
License: (c) (i) This work is licensed under a Creative Commons Attribution 4.0 International License. Read Full License 


\section{Abstract}

Background: The CRASH-3 trial provides a high level of evidence on the question whether to administer Tranexamic Acid (TXA) for Traumatic brain injury (TBI). For numerous other research questions, the available evidence will not correspond to such a level of evidence and will rely on observational evidence only. The development of methodological alternatives to analyze observational data is necessary. The Crash-3trial provided the opportunity to explore the effect of TXA on TBI mortality with two distinct causal inference methods using incomplete observational data.

Methods: Two causal inference techniques, inverse propensity weighting (IPW) and doubly robust method (DR), associated with machine learning method techniques to handle missing data, explored the effect of TXA administration on 30-day head injury related death expressed in registry data. The effect was expressed as Average Treatment Effect (ATE). TBI was defined as a head Abbreviated Injury Score $>2$. The hypothesis expected the results to concur with the results obtained with the CRASH-3 benchmark trial.

Results: Between September 2010 and February 2019, from a total of 20037 registry cases 8269 corresponded to the definition of TBI. A total of 683 received TXA and 7565 did not. The observed headinjury related 30-day hospital mortality rate in the group TXA was $30 \%(205 / 683)$ compared to $15 \%$ in the group no-TXA (1102/7565, $\mathrm{p}<0.001)$. Causal inference with the IPW approach indicates an ATE with a higher mortality after TXA independently of the approach applied to manage missing data (ATE 0.10 $(95 \%$ IC $[0.06,0.14])$ or $0.09(95 \%$ IC $[0.03,0.15]))$. ATE obtained with DR did not show any effect on mortality independently of the approach applied to missing data (ATE $-0.01(95 \%$ IC $[-0.05,0.03])$ or -0.01 $(95 \%$ IC $[-0.07,0.05]))$. No effect was observed in predefined subgroups.

Conclusions: This study demonstrated the feasibility to apply causal inference techniques in incomplete observational data. DR based on a stronger theoretical background compared to IPW, did not show a significant association of TXA administration with in-hospital mortality. This result provides a strong incentive to explore augmented causal inference techniques on incomplete observational data coupled with techniques to handle missing values.

\section{Background}

Severe traumatic brain injury (TBI) remains a major global public health challenge. The global incidence is expected to rise, due to increased road traffic in the developing world and a higher proportion elderly in all populations[1]. The CRASH-3 landmark trial examined the use of Tranexamic Acid (TXA) to tackle the challenge of TBI with exemplary methodological rigor[2]. The trial demonstrated no reduction in overall head injury-related 28-day mortality, but a reduction in the pre-specified subgroup of mild to moderate TBI (GCS 9-13).

Despite the conclusion of two recent studies[3, 4], one randomized, and a meta-analysis incorporating several other underpowered RCT's[5] the result of CRASH-3 remains the most reliable evidence on TXA 
use in TBI with a beneficial risk benefit ratio[6].

In contrast, to the administration for TXA in TBI, for many research questions, the medical community does not and will not dispose of results from prospective and randomized trials but rely on observational data only. Alternatives are needed to improve inference from observational data. Causal inference attempts to determine the independent influence of an effector as a component of a complex system. Causal inference from observational data differs from association by analyzing the response of an effector variable when a cause of the effect variable is changed. Methods from this family of approaches, for instance propensity weighting or matching, have been successfully applied by physics, climate research, econometrics and cognitive science[7, 8]. Augmented causal inference and related techniques appear more reliable than conventional observational and pathophysiological research [9] and help to develop more robust hypotheses for prospective research. Augmented Causal inference for observational data is not meant not become a substitute for randomized controlled trials but a useful addition to the methodological arsenal. To learn about and familiarize with this concept in reference to high level evidence such as provided by the CRASH-3 trial appears necessary.

Based on this rationale the present study investigated the capacity of two causal inference approaches (inverse propensity weighting and doubly robust method) combined with handling of missing data and to interpret the results into the context of the available clinical evidence[5]. The hypothesis expected the results to concur with the results obtained with the CRASH-3 benchmark trial.

\section{Methods}

This observational study is based on data from a prospective multicenter regional trauma registry, the TraumaBase@(TB). The registry disposes of algorithms for consistency and coherence and professional data monitoring. Data monitoring for the TraumaBase $\odot$ is assured by the Biostatistics Laboratory of Paris 7 ([10]).

\section{Setting and Cohort}

All consecutive trauma patients admitted to one of the 14 participating trauma centers were screened for inclusion. The appendix I provides the complete list variables that were recorded for each patient according to the revised Utstein major trauma template [11]. The trauma system in participating trauma centers have been previously described $[10,12,13]$; management was left to the discretion of the responsible physician based on national triage[14] and TBI management guidelines [15]. The corresponding Strobe checklist is provided in the appendix (I.a).

\section{Inclusion criteria}

The traumatic brain injury was defined as cerebral injury identified on a brain CT scan on admission corresponding to and Abbreviated Injury Score $>2$.

\section{Exclusion criteria}


Patients were excluded if their age was < 16 years old or the patient was admitted to a center with less than 20 traumatic brain injuries (TBI) over the entire inclusion period.

\section{Administration of Tranexamic Acid (TXA)}

TXA administration followed the CRASH-2 protocol, an intravenous dose of 1 gram over 10 minutes, followed immediately by a second intravenous dose of 1 gram over 8 hours. For the purpose of the study, the authors considered, that all administration occurred within three hours of injury.

\section{Data extraction}

A complete list of collected data can be found in the Appendix I.b).

\section{Analysis}

The objective was to evaluate the effect of TXA on 30-day head-injury related mortality in a cohort of traumatic brain injured patients by causal inference. The study cohort was stratified into predefined subgroups in accordance with the CRASH-3 trial: GCS 9-12 and less than 8 and pupil anomaly. Other outcomes reported are all cause 30-day mortality and all-cause mortality as well as all head-injury related deaths to allow comparison with other studies. Data are presented as absolute count and percentages (n, \%) for categorial and median (interquartile range) for numerical data. Categorical data were compared using a Chi-2 or Fisher Exact Test, numerical data using a Mann-Whitney Test. The analysis was performed on the whole cohort stratified into "TXA administration" and "no TXA administration". A p value $<0.05$ was considered as significant. The statistical package $R$ version 3.6.2 was used for the entire analysis of this study (R Core Team (2019). R: A language and environment for statistical computing. $R$ Foundation for Statistical Computing Vienna, Austria) and associated following packages (cite packages if needed)

\section{Adopted Causal Inference approach}

A detailed description and essential theoretical concepts can be found in the appendix section II.a-X following.

a) Exposure variable and outcome criteria

Traumatic Brain Injury (TBI) was defined as stated above. The exposure variable was administration of tranexamic acid (TXA) during prehospital care or on admission to the resuscitation room and considered to have occurred within three hours of the initial trauma. TXA administration followed the CRASH-2 protocol. The main outcome of interest was all cause in-hospital mortality. Patients who died within 24 hours were retained in the analysis to minimize survivor bias.

b) Measure of Impact

The measure of impact was the average treatment effect (ATE) and corresponded to the difference in mortality between TBI patients exposed to TXA compared to patients not exposed. The ATE and the 
corresponding $95 \%$ confidence interval $(95 \% \mathrm{Cl})$ were calculated for the entire cohort and pre-defined subgroups: TBI severity (mild/moderate, severe) and pupil reactivity (normal, reactive, non-reactive). ATE corresponds to the estimation of the average effect of the treatment to reduce mortality, expressed in \% points difference between the two groups (see appendix for the CRASH-3 results expressed as ATE). ATE was considered in favour of a causal relationship if the $95 \% \mathrm{Cl}$ did not include 0 . All $95 \% \mathrm{Cl}$ were calculated with a non-parametric Bootstrap method.

c) Identification of confounding factors

Potential confounders were identified by a Delphi process consulting with a group of 10 experts in TBI[16]. Pre-intervention (prior to the administration of TXA) variables identified by the Delphi process concerned factors that would influence the clinician to administer TXA (e.g. haemorrhage, see Fig. 1). In the final model (see section doubly robust method) all variables associated to the severity of the TBI and hospital mortality (e.g. GCS) as well as criteria associated with the treatment administration were mapped with the program dagitty ${ }^{17}$ into a Directed Acyclic Graph (DAG, Fig. 1) as recommended ${ }^{18}$.

d) Balancing

In order to estimate the average treatment effect (ATE), deconfounding or balancing of the groups was achieved by reweighting observations and consequently comparing the standardized mean differences; the differences were not to exceed a $20 \%$ threshold.

e) Effect estimation

The ATE was estimated by

1) Inverse propensity score weighting, requiring a model capturing the confounding factors to debias the treatment assignment and

2) Doubly robust approach requiring a model capturing the confounding factors to debias the treatment assignment and a model that relates the confounding factors to the outcome, the outcome model.

For more details, refer to Appendix II.a-h

e) Sensitivity analysis (see Appendix VI)

After estimation of the treatment effects, a sensitivity analysis was performed to assess how much the final results would change if one or more of the working assumptions such as unconfoundedness and expert based plausibility were violated.

f) Management of missing data

Missing data were managed with two distinct methods (Appendix III): 
- MICE, multiple imputation with chained equations

- MIA, missing incorporated in attributes

MICE, and multiple imputation in general, is a probabilistic imputation method exploiting the correlation between different variables replacing missing values with several plausible values [17]. MIA uses random forests algorithms to learn complex relationships between predictive factors and treatment allocation/outcome and additionally about the information in the missing values patterns in the data.

\section{Results}

\section{Cohort and propensity score weighting}

Between September 2010 and February 2019, a total of 20037 separate trauma cases have been incorporated into the Traumabase Registry in 14 participating centers (Flowchart in Appendix I.c). Among this sample, 8269 corresponded to the definition of TBI. A total of 683 received TXA and 7565 did not receive TXA. Table 1 illustrates that the clinical characteristics of the two groups before propensity score adjustment differed significantly. Patients with administration of TXA were more severely injured, showed higher SAPS II scores, and were more often in shock or required more often neurosurgery and neurocritical care.

Table 1, cohort characteristics 


\begin{tabular}{|c|c|c|c|}
\hline Variable & $\begin{array}{l}\text { TXA group } \\
(n=683)\end{array}$ & $\begin{array}{l}\text { Control group } \\
(n=7565)\end{array}$ & $\mathrm{p}$ \\
\hline Gender $=$ male, $n(\%)$ & $460(67)$ & $5817(77)$ & $\dot{0.001}$ \\
\hline Age (years) & 39 [26-55] & $40[26-58]$ & 0.099 \\
\hline$<25, n(\%)$ & $139(20)$ & $1654(22)$ & 0.004 \\
\hline $25-44, n(\%)$ & $275(40)$ & $2551(34)$ & 0.004 \\
\hline $45-64, n(\%)$ & $172(25)$ & $2000(27)$ & 0.004 \\
\hline$\geq 65, n(\%)$ & $97(14)$ & $1338(18)$ & 0.004 \\
\hline Anticoagulant therapy, $n(\%)$ & $24(4)$ & $395(5)$ & 0.084 \\
\hline Antiplatelet therapy, $n$ (\%) & $33(5)$ & $363(5)$ & 0.703 \\
\hline Initial GCS in pre-hospital phase & $7[3-14]$ & $13[6-15]$ & $\begin{array}{l}< \\
0.001\end{array}$ \\
\hline Initial GCS = 3, n (\%) & $227(34)$ & $1042(14)$ & $\begin{array}{l}< \\
0.001\end{array}$ \\
\hline $\begin{array}{l}\text { Initial GCS }=3 \text { or bilateral mydriasis in pre-hospital } \\
\text { phase, } n(\%)\end{array}$ & $240(36)$ & $1146(16)$ & $\begin{array}{l}<.001 \\
0.001\end{array}$ \\
\hline \multicolumn{4}{|l|}{ AIS } \\
\hline Head & 3 [3-5] & $3[2-5]$ & \multirow{2}{*}{$\begin{array}{l}<.001 \\
0.001\end{array}$} \\
\hline Face & $0[0-2]$ & $0[0-1]$ & \\
\hline \multirow[t]{2}{*}{ External } & $0[0-0]$ & $0[0-0]$ & $\begin{array}{l}<.001 \\
0.01\end{array}$ \\
\hline & & & 0.193 \\
\hline ISS & 38 [29-50] & 21 [13-29] & $\begin{array}{l}<.001 \\
0.001\end{array}$ \\
\hline \multicolumn{4}{|l|}{ Pupil anomaly in pre-hospital phase } \\
\hline Anisocoria, $n(\%)$ & $93(14)$ & $689(9)$ & \multirow{2}{*}{$\begin{array}{l}< \\
0.001\end{array}$} \\
\hline Bilateral mydriasis, $n(\%)$ & $163(24)$ & $605(8)$ & \\
\hline None, $n(\%)$ & $418(61)$ & $6142(81)$ & 0.001 \\
\hline \multirow[t]{2}{*}{ Not specified, $n(\%)$} & $9(1)$ & $129(2)$ & $<.001$ \\
\hline & & & $<.001$ \\
\hline
\end{tabular}


Osmotherapy in pre-hospital phase

Mannitol, $n$ (\%)

$122(18)$

$822(11)$

$<$

Hypertonic saline serum, $n$ (\%)

$20(3)$

None, $n(\%)$

$113(17)$

$134(2)$

$514(7)$

0.001

$<$

Improvement of pupil anomaly after osmotherapy

$$
\begin{aligned}
& \text { Yes, } n(\%) \\
& \text { No, } n(\%)
\end{aligned}
$$

$142(21)$

$346(5)$

$765(10)$

$<$

0.001

$<$

0.001

Blood pressure in pre-hospital phase

Systolic

Diastolic

105 [80-125]

$130[116-$

148]

$<$

$62[45-80]$

$80[68-90]$

$<$

0.001

Heart rate in pre-hospital phase

108 [78-127]

$87[72-101]$

$<$

0.001

Shock index in pre-hospital phase

0.96 [0.69-1.3]

$0.66[0.53-$

$0.81]$

$<$

0.001

Minimal Sp02 in pre-hospital phase

95 [87-99]

97 [95-99]

$<$

0.001

Activation of hemorrhagic shock procedure, $n(\%)$

$96(14)$

$1261(17)$

0.087

Cardiac arrest in pre-hospital phase, $n$ (\%)

$131(19)$

$358(5)$

$<$

0.001

Cristalloid volume $(\mathrm{mL})$

$1000[750-$

1500]

$500[500-$

1000]

$<$

0 [0-500]

$0[0-0]$

$<$

Colloid volume $(\mathrm{mL})$

$397(58)$

$1064(14)$

$<$

Vasopressor therapy, $n$ (\%)

13 [3-15]

15 [13-15]

$<$

GCS at hospital admission

0.001

0.001

Pupil anomaly at hospital admission

Anisocoria, $n(\%)$

$93(14)$

$689(9)$

$<$

0.001 
Bilateral mydriasis, $n$ (\%)

$163(24)$

$605(9)$

$<$

None, $n(\%)$

$418(61)$

$6142(81)$

0.001

$<$

0.001

\section{Osmotherapy at hospital admission}

Mannitol, $n$ (\%)

Hypertonic saline serum, $n(\%)$

Aucune, $n(\%)$
$122(18)$

$127(19)$

$434(64)$
$922(12)$

599 (8)

$6044(80)$
$<$

0.001

$<$

0.001

0.001

IGS ||

$60[45-75]$

$30[17-49]$

$<$

0.001

Maximal Pulsatility index with transcranial Doppler

Traumatic brain injury on brain scan

$1.2[0.9-1.6]$

$1.1[0.9-1.4]$

0.003

$479(70)$

$4999(66)$

0.035

Inverse propensity weighting (IPW) generated two comparable groups as demonstrated in Fig. 2 . The distribution of missing data was equally weighted to acknowledge for distinct distribution in each group (see Appendix IV).

\section{Main outcome criterion}

Before application of the causal inference approach the observed head-injury related 30-day mortality in the group TXA was $30 \%(205 / 683)$ compared to $15 \%$ in the group no-TXA (1102/7565), $p<0.001$. Figure 3 illustrates results obtained with causal inference for the main outcome. The Average Treatment Effect (ATE) indicates the mortality difference in the group TXA versus the group without TXA. Causal inference according to IPW indicates an ATE suggesting an objective association with a higher 30-day head-injury related mortality after TXA administration independently of the approach applied to estimate missing data (ATE MICE: 0.10 (95\% IC [0.06, 0.14]); ATE MIA: 0.09 (95\% IC [0.03, 0.15])). Results obtained with doubly robust method did not show any effect of the treatment on 30-day head-injury related mortality (ATE MICE: -0.01 (95\% IC [-0.05, 0.03]); ATE MIA: -0.01 (95\% IC [-0.07, 0.05])).

\section{Subgroup Analysis}

Table 2 exposes the results after stratification of the two groups according to TBI severity (severe GCS $\leq$ 8, moderate/mild GCS 9-15), pupil reaction (reactive versus non-reactive). IPW estimates an ATE in favour of an increased mortality in mild TBI patients (mild/moderate GCS or reactive pupils) no matter which imputation technique is applied compared to DR. For severe patients (severe GCS or non-reactive pupils) both approaches, IPW and DR, agree on a non-significant effect, independently of the imputation technique. 
The appendix provides all-cause mortality and 30-day all-cause mortality and all head-injury related mortality and the corresponding ATE including subgroups. Independently of the definition applied, no mortality definition and in no subgroup, we could show a causal relationship between TXA and mortality.

Table 2

, Head injury related $30 d$ death stratified into subgroups according to GCS and pupil response

\begin{tabular}{|lll|}
\hline Inverse propensity weighting & ATE with MICE imputation & ATE with MIA estimation \\
\hline GCS $=\mathbf{9 - 1 5}$ & $0.10(95 \%$ IC $[0.02,0.18])$ & $0.07(95 \%$ IC $[0.03,0.11])$ \\
\hline GCS $\leq \mathbf{8}$ & $0.07(95 \%$ IC $[-0.07,0.21])$ & $0.07(95 \%$ IC $[-0.01,0.15])$ \\
\hline Reactive pupils & $0.13(95 \%$ IC $[0.07,0.19])$ & $0.11(95 \%$ IC $[0.05,0.17])$ \\
\hline Non reactive pupils & $0.01(95 \%$ IC $[-0.28,0.30])$ & $0.01(95 \%$ IC $[-0.11,0.13])$ \\
\hline Doubly robust method & ATE with MICE imputation & ATE with MIA estimation \\
\hline GCS $=9-15$ & $0.02(95 \%$ IC $[-0.08,0.12])$ & $0.00(95 \%$ IC $[-0.06,0.06])$ \\
\hline GCS $\leq \mathbf{8}$ & $-0.02(95 \%$ IC $[-0.16,0.12])$ & $-0.01(95 \%$ IC $[-0.05,0.03])$ \\
\hline Reactive pupils & $0.05(95 \%$ IC $[-0.01,0.11])$ & $0.00(95 \%$ IC $[-0.06,0.06])$ \\
\hline Non reactive pupils & $-0.11(95 \%$ IC $[-0.38,0.16])$ & $-0.01(95 \%$ IC $[-0.07,0.05])$ \\
\hline
\end{tabular}

\section{Discussion}

This study applied two causal inference techniques, inverse propensity weighting (IPW) and doubly robust method (DR), combined with handling of missing data to estimate the effect of TXA on patients with TBI from observational data. Based on the present large observational database, IPW seems to overestimate a harmful effect of TXA on mortality when compared to the CRASH-3 reference. By contrast, the estimation based on Doubly Robust (DR) suggests that TXA administration after TBI does not exert any effect on mortality.

How do these results relate to the available evidence? First, the present study truly does not compare to the available prospective evidence. Any evaluation between the presented results and prospective evidence in particular CRASH-3 only serves the purpose to appreciate the performance of the deployed statistical (or analysis) techniques. Second, prospective studies diverge in crucial points such as power, outcome criteria (28-day mortality versus 30-day or all-cause mortality), pre-hospital or intrahospital TXA administration, excluding extracranial hemorrhage, administration protocols.

The results for ATE in \% of risk of head injury related death for the Doubly Robust method ranged from a $7 \%$ decrease to a $5 \%$ increase depending on the method to estimate the missing values (MICE of MIA) and $3-15 \%$ increase with IPW. In comparison, CRASH-3 risk of head injury related death in the overall cohort ranged from a $14 \%$ decrease to a $2 \%$ increase with a mean of a $1.3 \%$ decrease[2]. Rowell at al [3] 
showed a range from a $8 \%$ decrease for 28 -day mortality to a $2 \%$ increase, with a mean of $3 \%$ decrease. The meta-analysis[5] concluded to a $3 \%$ decrease to a $1 \%$ increase in overall mortality.

The main challenge in any causal inference approach from observational data is the control of confounders. Bossers et al., a registry based observational study, showed an increase in 28-day mortality concurring with the ATE estimation by IPW. Bossers et al. established the association through unadjusted logistic regression, then adjusted for confounders in contrast the present study based on expert knowledge and directed acyclic graphs as recommended[9]. The results discordant from CRASH-3 obtained by Bossers et al. and IPW may however pertain to the same difficulty to obtain sufficient control for confounding.

When comparing observational data from two very disparate groups, standard propensity scores methods tend to under-correct for the observed difference, either due to model misspecification (in the case of logistic regression) or insufficient sample size (in case of random forest regression). In consequence the estimation of the treatment effect becomes erroneous. In the present case IPW seems not to have sufficiently corrected for the treatment bias, probably because it struggles to achieve sufficient control of confounders. IPW and DR require both a sufficient knowledge of all confounding factors. DR however provides better control of potential bias and smaller variability than IPW, integrating at the same time a prediction of mortality and of treatment allocation. This dual modelling of mortality and treatment allocation optimally exploits the available data and protects against misspecification of either one of the models, making it more robust than IPW. Furthermore, the flexibility of random forests in the doubly robust method engenders a more powerful model capturing complex relationships and is suited for application to a large cohort. The first take-away from the present paper is therefore when employing causal inference, DR is preferrable to IPW. This study also shares an important innovation, since it is the first to combine DR with two advanced methods to handle missing data and both generate concordant results.

Trials in critical care in the last fifteen years often produced negative results and were regularly underpowered to detect frequently unattainable outcome targets [7]. Mortality as the most certified outcome criterion often falls short to picture heterogenous effects of complex interventions in complex disease [8]. Furthermore, RCTs consume precious human, financial, organizational and time resources. Benchmark trials such as CRASH-3 result from exemplar international research efforts, not applicable or reproducible to many research questions. Not only because of resource constraints, but the necessary recruitment remains unobtainable in an appropriate timeframe. Recruitment is not facilitated by the increasingly small marginal benefits bestowed by ever more complex interventions. Despite a strong rationale, CRASH-3 required more than 12000 patients.

Augmented causal inference does not aspire to become a substitute for randomized controlled trials but is capable to upgrade conventional observational in particular in the era of big data and physiological research and to provide a better rationales for RCTs [9]. The approach could become a reference to prepare RCTs, explore the association of different interventions or bundles in different subgroups. This 
customized preparation would funnel research resources to the most promising RCTs. For this reason, the results of this study using augmented causal inference appear promising and should be further explored. An association of prospective, randomized data and parallel augmented causal inference on observational dataset could be feasible.

The study imparts specific limitations. The inclusion period spans from 2010 to 2019, over this long period it is likely that management and epidemiology have evolved. The study group considered all TXA to be administered within three hours of injury. Furthermore, TXA was administered for suspected hemorrhage and not TBI, making the effect on isolated TBI difficult to assess; the association of hemorrhage and TBI might have affected the outcome prediction, although this was accounted for in the model. Among the patients included with TBI, 842 presented with severe acute hemorrhage (received at least 4 red blood cell packs within the first six hours). The choice of confounders and treatment allocation variables was based on expert advice and could be a possible source of bias. Even experts may fail to perceive alternative explicative patterns and risk to appreciate only the patterns they know and are prone to inherent cognitive bias. Despite use of a Delphi and DAG to map possible confounders to account for these in the final model, some variables might still escape sufficient control. Collected data can be imprecise (for example blood pressure measurements) and are only a fragmented surrogate for a complex physiological process (a few blood pressure measurements at various time points versus continuous data). Missing data constitute an inherent limitation of any work based on off-the-shelf observational data; missingness of data is impossible to prevent in particular in registry data and all the more so in a clinical context of emergency. Fully aware of this intrinsic limitation of registry data, the study group set out to purposefully integrate and advance management of missing data, testing two different methods for imputation of missing data. With all these imperfections, control for confounding in causal inference of observational data remains a formidable challenge. Future studies need to address this challenge including the quality and mapping capacity of observational data. Finally, the chosen threshold of a Mean Standardized Difference of $20 \%$ as accepted might seem important, in particular since mortality differences between the groups are $<20 \%$.

\section{Conclusion}

This study explored the feasibility to estimate the effect of TXA administration on TBI in relationship to available prospective evidence, in particular an international benchmark trial combined with an innovative approach to handle missing data. In comparison to the IPW approach, the doubly robust method provides a better estimate of the effect of TXA. Possibly IPW provides insufficient control of confounding. This result provides a strong incentive to explore augmented causal inference techniques on observational data as an alternative to situations where randomized controlled trials are impossible or in the preparation of RCTs.

\section{Abbreviations}


ATE= Average Treatment Effect; CT = Computer Tomography; DAG= Directed Acyclic Graph; GCS= Glasgow Coma Scale; DR=Doubly Robust Method; MIA= Missing Incorporated in attributes; MICE= Multiple imputation with chained equations; MSD = Mean Standardized Difference; IPW=Inverse Propensity Weighting; RT = Randomized Controlled TBI= Traumatic Brain Injury; TXA=Tranexamic Acid

\section{Declarations}

\section{Ethics approval and consent to participate}

This registry has obtained approval from the Institutional Review Board (Comité de Protection des Personnes, Paris VI and Clermont-Ferrand) from the Advisory Committee for Information Processing in Health Research (Comité Consultatif Pour le Traitement de l'information en matière de recherche dans le domaine de la santéCCTIRS, 11.305bis) and from the National Data Protection Agency (Commission Nationale de l'Informatique et des Libertés CNIL, 911461), waiving the need for informed consent.

\section{Consent for publication}

All participating authors provided consent for publication

\section{Availability of data and material}

All data and scripts in this study are available upon request.

\section{Competing interests}

TG declares having received honoraria from Laboratoire du Biomédicament Français in the past with relation to the present work. AH declares having received honoraria from Laboratoire du Biomédicament Français and Edwards company in the past. The others authors have no conflict to declare.

\section{Funding}

The Traumabase registry is in part funded by the Regional Health Authority lle de France and Est (Agence Regionale de Sante, ARS). The Regional Health Authority had no role in the conception, collection interpretation or analysis of the data.

\section{Authors' contributions}

Concept, Design: IM, TG, JDM

Acquisition, Analysis, Interpretation of data: All authors

Statistical Analysis: IM, JJ, JPN

Drafting of manuscript: TG, IM, AD 
Critical revision of manuscript: All authors have approved and read the manuscript

\section{Acknowledgements}

We would like to thank all members of the Traumabase study group for their continued commitment. Without their daily efforts studies like the present one would be impossible.

\section{References}

1. James SL, Theadom A, Ellenbogen RG, Bannick MS, Montjoy-Venning W, Lucchesi LR, et al. Global, regional, and national burden of traumatic brain injury and spinal cord injury, 1990-2016: a systematic analysis for the Global Burden of Disease Study 2016. The Lancet Neurology. 2019;18:56-87.

2. CRASH-3 trial collaborators. Effects of tranexamic acid on death, disability, vascular occlusive events and other morbidities in patients with acute traumatic brain injury (CRASH-3): a randomised, placebo-controlled trial. Lancet. 2019;394:1713-23.

3. Rowell SE, Meier EN, McKnight B, Kannas D, May S, Sheehan K, et al. Effect of Out-of-Hospital Tranexamic Acid vs Placebo on 6-Month Functional Neurologic Outcomes in Patients With Moderate or Severe Traumatic Brain Injury. JAMA. 2020;324:961.

4. Bossers SM, Loer SA, Bloemers FW, Den Hartog D, Van Lieshout EMM, Hoogerwerf N, et al. Association Between Prehospital Tranexamic Acid Administration and Outcomes of Severe Traumatic Brain Injury. JAMA Neurol. 2020. doi:10.1001/jamaneurol.2020.4596.

5. Lawati KA, Sharif S, Maqbali SA, Rimawi HA, Petrosoniak A, Belley-Cote EP, et al. Efficacy and safety of tranexamic acid in acute traumatic brain injury: a systematic review and meta-analysis of randomized-controlled trials. Intensive Care Med. 2021;47:14-27.

6. Maas AIR, Steyerberg EW, Citerio G. Tranexamic acid in traumatic brain injury: systematic review and meta-analysis trumps a large clinical trial? Intensive Care Med. 2020;:s00134-020-06305-x.

7. Harhay MO, Wagner J, Ratcliffe SJ, Bronheim RS, Gopal A, Green S, et al. Outcomes and Statistical Power in Adult Critical Care Randomized Trials. Am J Respir Crit Care Med. 2014;189:1469-78.

8. Dreyfuss D. Faut-il continuer à faire des études randomisées ? Revue des Maladies Respiratoires. 2005;22:381-5.

9. Lederer DJ, Bell SC, Branson RD, Chalmers JD, Marshall R, Maslove DM, et al. Control of Confounding and Reporting of Results in Causal Inference Studies. Guidance for Authors from Editors of Respiratory, Sleep, and Critical Care Journals. Annals ATS. 2019;16:22-8.

10. Gauss T, Ageron F-X, Devaud M-L, Debaty G, Travers S, Garrigue D, et al. Association of Prehospital Time to In-Hospital Trauma Mortality in a Physician-Staffed Emergency Medicine System. JAMA Surg. 2019;154:1117-24.

11. Ringdal KG, Coats TJ, Lefering R, Di Bartolomeo S, Steen PA, Røise O, et al. The Utstein template for uniform reporting of data following major trauma: a joint revision by SCANTEM, TARN, DGU-TR and 
RITG. Scand J Trauma Resusc Emerg Med. 2008;16:7.

12. Hamada SR, Gauss T, Duchateau F-X, Truchot J, Harrois A, Raux M, et al. Evaluation of the performance of French physician-staffed emergency medical service in the triage of major trauma patients. J Trauma Acute Care Surg. 2014;76:1476-83.

13. Hamada SR, Delhaye N, Degoul S, Gauss T, Raux M, Devaud M-L, et al. Direct transport vs secondary transfer to level I trauma centers in a French exclusive trauma system: Impact on mortality and determinants of triage on road-traffic victims. PLoS One. 2019;14:e0223809.

14. Riou B, Thicoipe M, Atain-Kouadio P. Comment évaluer la gravité? Actual En Réanimation Préhospitalière Traumatisé Grave. 2002;:115-28.

15. Geeraerts T, Velly L, Abdennour L, Asehnoune K, Audibert G, Bouzat P, et al. Management of severe traumatic brain injury (first 24hours). Anaesth Crit Care Pain Med. 2018;37:171-86.

16. Jones J, Hunter D. Qualitative Research: Consensus methods for medical and health services research. BMJ. 1995;311:376-80.

17. Buuren $S$ van. Flexible imputation of missing data. Second edition. Boca Raton: CRC Press, Taylor and Francis Group; 2018.

\section{Figures}




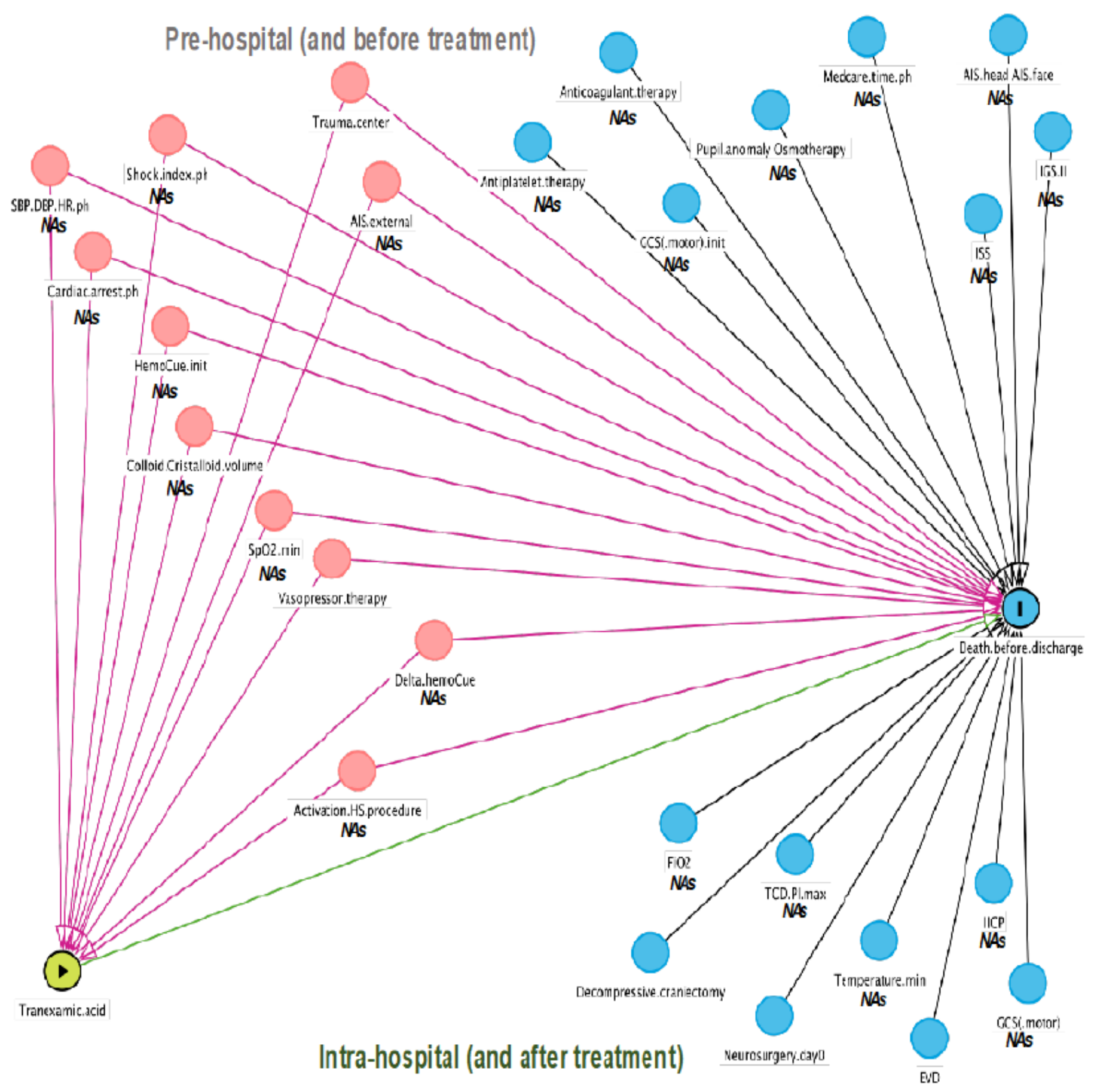

\section{Figure 1}

Directed Acyclic Graph (DAG): a) pre-intervention variables associated with the decision to administer Tranexamic Acid (TXA) in purple; arrows point toward TXA administration or death if the same variables are associated with death. b) Explicative variables associated with the main outcome death (blue arrows) independent from treatment administration. ph=prehospital; init=initial, SBP/DBP=systolic/diastolic blood pressure; $\mathrm{HR}=$ heart rate; $\mathrm{SpO2}$. $\mathrm{min}=$ minimal peripheral oxygen saturation in prehospital phase; HemoCue=capillary hemoglobin concentration; delta HemoCue=difference prehospital and admission capillary hemoglobin; Activation HS procedure=activation of hemorrhagic shock procedure; 
TCD.PI.max=maximal pulsatility index measured with transcranial doppler, EVD=external ventricular drain, IICP=at least one episode of increased PI, GCS=Glasgow Coma Scale, ISS=Injury Severity Score, AIS=Abbreviated Injury Score, IGS.II=Simplified acute physiology score

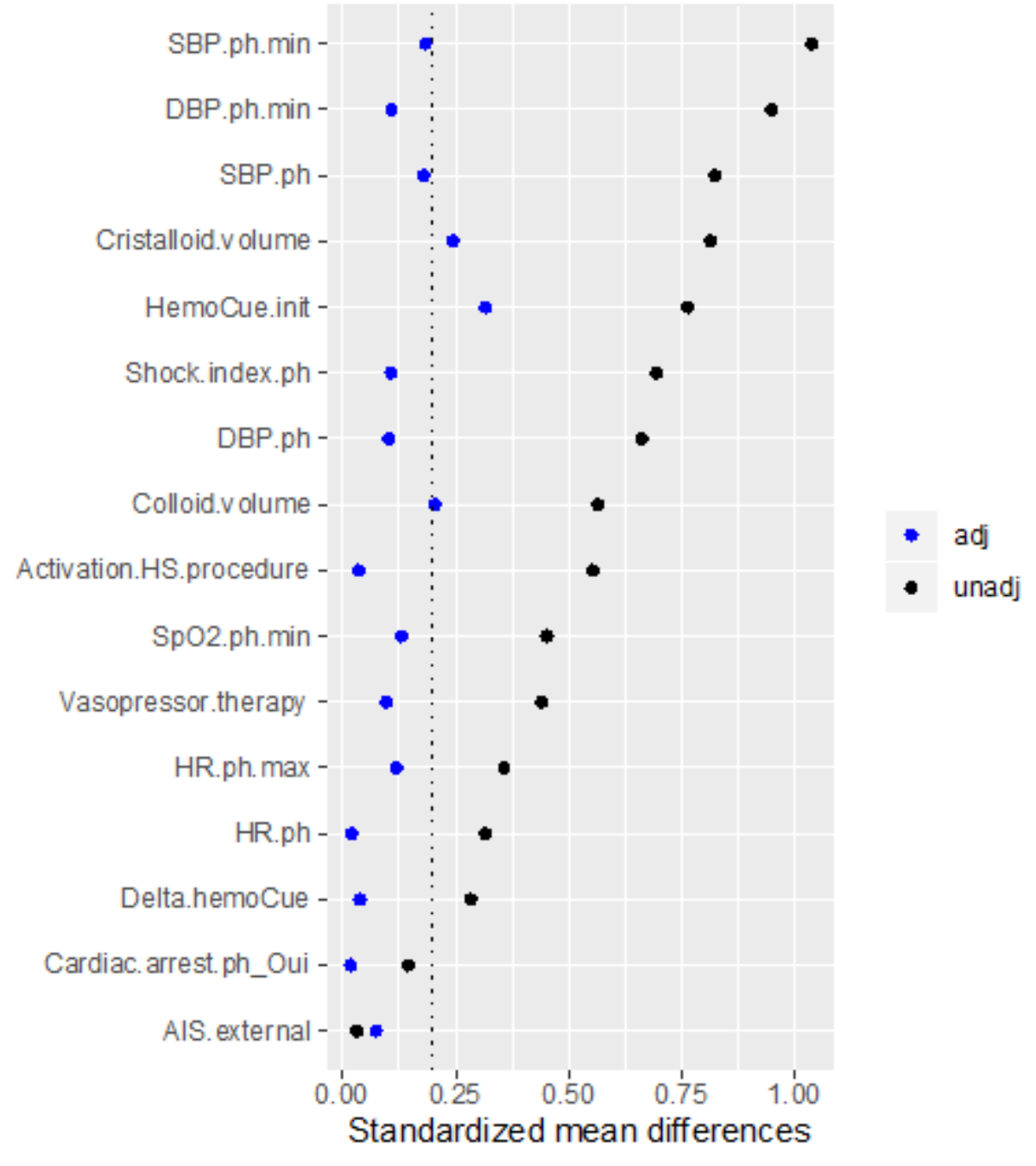

\section{Figure 2}

Effect of inverse propensity weighting (IPW) on Mean Standardized Differences (MSD) in absolute values. Black dots represent absolute values of the MSD before inverse propensity weighting. Blue dots represent absolute values of the MSD after inverse propensity weighting. All confounding factors demonstrated an MSD < 20\% after IPW except for capillary hemoglobine (=Hemocue); blue points =adjusted after IPW, black points = unadjusted before IPW 


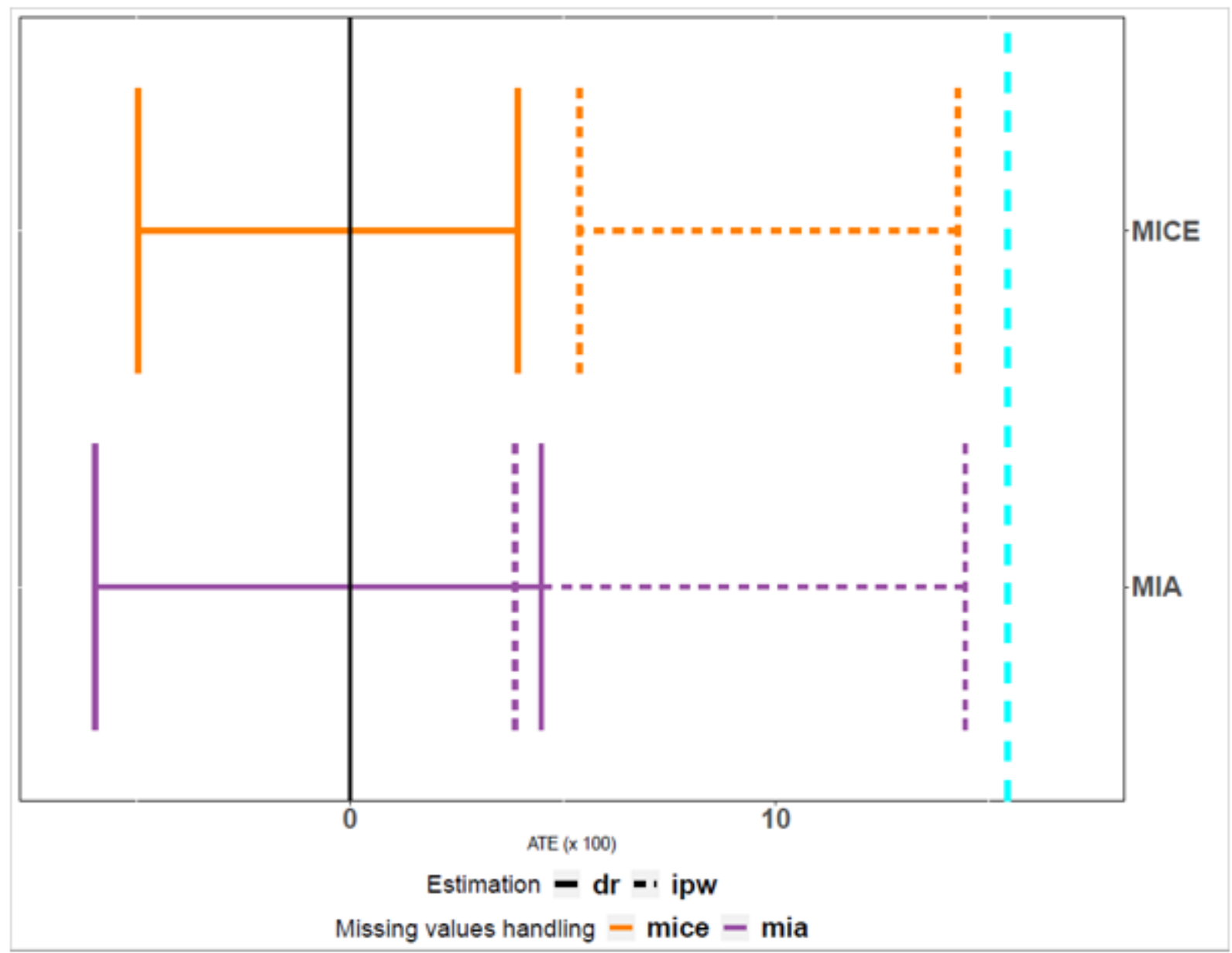

\section{Figure 3}

Estimation of ATE (Average treatment effect) on 30-day head injury related death after application of causal inference with inverse propensity weighting (IPW) and doubly robust method (DR). In dotted lines estimation by IPW, in uninterrupted lines estimation by DR; in orange imputation of missing data with MICE; in purple estimation of missing data with MIA. In cyan dashed line the unadjusted ATE (without reweighting of the treatment groups). A negative ATE excluding zero favours the TXA group over the group without TXA. (ATE=average treatment effect; $d r=$ doubly robust method; ipw=inverse propensity weighting, $\mathrm{MICE}=$ multiple imputation with chained equations; $\mathrm{MIA}=$ missing incorporated in attributes)

\section{Supplementary Files}

This is a list of supplementary files associated with this preprint. Click to download.

- AppendixTBITXA04052021.docx 